Meta

Journal des traducteurs

Translators' Journal

\title{
De la traduction juridique à la jurilinguistique : la quête de l'équivalence
}

\section{Jean-Claude Gémar}

Volume 60, numéro 3, décembre 2015

URI : https://id.erudit.org/iderudit/1036139ar

DOI : https://doi.org/10.7202/1036139ar

Aller au sommaire du numéro

\section{Éditeur(s)}

Les Presses de l’Université de Montréal

\section{ISSN}

0026-0452 (imprimé)

1492-1421 (numérique)

Découvrir la revue

Citer cet article

Gémar, J.-C. (2015). De la traduction juridique à la jurilinguistique : la quête de l'équivalence. Meta, 60(3), 476-493. https://doi.org/10.7202/1036139ar

\section{Résumé de l'article}

Dans un pays où règnent un bilinguisme officiel et un bijuridisme d’État, la traduction n'est jamais loin et l'opération traduisante passe alors obligatoirement par la comparaison des droits. Ces deux activités sont plus étroitement liées qu'on ne le pense. Aussi n'est-il guère étonnant que, parmi les comparatistes, on trouve des spécialistes avertis de la traduction juridique et que des traducteurs soient formés à la jurilinguistique. Le Canada s'est illustré de longue date dans ces deux domaines. En deux siècles de pratique, il a décliné toute la gamme de procédés et méthodes de traduction de ses textes juridiques. À l'origine, littérale et servile, la manière de traduire est aujourd'hui plus libre. L'équivalence - dont nous évoquons la saga - est devenue " fonctionnelle "; la corédaction (bilingue anglais-français) est apparue dans le processus de production des lois. Mais l'équivalence des textes n'en est pas moins problématique et la question de l'interprétation du texte, traduit ou corédigé, n'est pas tranchée pour autant. En droit, l'opération traduisante requiert le savoir-faire du jurilinguiste pour produire l'équivalence " fonctionnelle » des textes, soit un rapport harmonieux entre la lettre et l'esprit du message que porte le droit. On avancera que le traducteur-jurilinguiste peut produire, au terme de son analyse, la « haute " traduction que, selon R. Sacco, seul un comparatiste peut produire. 


\title{
De la traduction juridique à la jurilinguistique: la quête de l'équivalence
}

\author{
JEAN-CLAUDE GÉMAR \\ Université de Montréal, Montréal, Canada \\ jean-claude.gemar@umontreal.ca
}

\section{RÉSUMÉ}

Dans un pays où règnent un bilinguisme officiel et un bijuridisme d'État, la traduction n'est jamais loin et l'opération traduisante passe alors obligatoirement par la comparaison des droits. Ces deux activités sont plus étroitement liées qu'on ne le pense. Aussi n'est-il guère étonnant que, parmi les comparatistes, on trouve des spécialistes avertis de la traduction juridique et que des traducteurs soient formés à la jurilinguistique. Le Canada s'est illustré de longue date dans ces deux domaines. En deux siècles de pratique, il a décliné toute la gamme de procédés et méthodes de traduction de ses textes juridiques. À l'origine, littérale et servile, la manière de traduire est aujourd'hui plus libre. L'équivalence - dont nous évoquons la saga - est devenue «fonctionnelle»; la corédaction (bilingue anglais-français) est apparue dans le processus de production des lois. Mais l'équivalence des textes n'en est pas moins problématique et la question de l'interprétation du texte, traduit ou corédigé, n'est pas tranchée pour autant. En droit, l'opération traduisante requiert le savoir-faire du jurilinguiste pour produire l'équivalence «fonctionnelle» des textes, soit un rapport harmonieux entre la lettre et l'esprit du message que porte le droit. On avancera que le traducteur-jurilinguiste peut produire, au terme de son analyse, la «haute» traduction que, selon R. Sacco, seul un comparatiste peut produire.

\begin{abstract}
In any country where official bilingualism is coupled with State bijuridism, translation is, of necessity, not far behind, and has to be performed through a comparative law process. These two activities are more closely related than is generally thought. It is not surprising to find among comparatists legal specialists who are knowledgeable about legal translation, while translators are trained jurilinguists. Canada has long stood out as a leader in these fields. Over two centuries, the country has applied a comprehensive range of procedures and methods to translating legal texts. Originally literal and slavish, today's translations are freer. Equivalence, whose history is summarized in the article, has become "functional," and codrafting (bilingual English and French) has developed into a method for preparing bilingual legislation. However, textual equivalence is still problematic and the issue of interpretation of legal texts, whether translated or codrafted, is far from settled. In the legal field, translation demands jurilinguistic know-how in order to produce a "functionally" equivalent target text, meaning a harmonious relationship between the letter and the spirit of the legal message. I argue that translator-jurilinguists, upon completing their analysis, can produce the "alta traduzione" that, according to R. Sacco, only a comparative lawyer can achieve.
\end{abstract}

\section{MOTS CLÉS/ KEYWORDS}

traduction juridique, jurilinguistique, droit, bilinguisme, équivalence fonctionnelle legal translation, jurilinguistics, law, bilingualism, functional equivalence 


\section{Introduction : aux sources de la jurilinguistique}

Si, comme le pensait le Montesquieu de L'Esprit des loix, les lois éclairent l'histoire d'un pays, celles du Canada le démontrent clairement, dans la lettre comme dans l'esprit. Ce «pays de traducteurs» (Vinay 1978: 22) est passé du stade d'une traduction servile, lourde et maladroite, des textes de droit des débuts de la colonisation britannique (1763), à «l'équivalence fonctionnelle» (Pigeon 1982: 271), au xx siècle, et, enfin, à l'étape ultime qu'est la «corédaction» des lois de l'État fédéral canadien dans le dernier quart du $\mathrm{xx}^{\mathrm{e}}$ siècle.

L'usage de traduire des textes de droit remonte aux lendemains de la Conquête (1759-1760), lorsque le nouveau gouvernement eut à communiquer à la population française, à partir de 1763, les lois, décrets, actes et autres textes juridiques qu'il produisait en anglais. Les mauvaises habitudes et les travers dénoncés par des générations de traducteurs et de linguistes datent de cette époque. Ils ont été reconduits dans l'Acte de l'Amérique du Nord britannique (1867) et ont perduré jusque dans les années 1960-1970 lorsque l'État canadien a commencé à instaurer des méthodes de production de ses textes juridiques visant à en améliorer la qualité. Le Canada fit alors un grand bond en avant avec la corédaction de ses lois, et la «jurilinguistique» - fille de la traduction et du droit - a fait son apparition. Depuis, l'exemple canadien s'est répandu, servant de modèle à de nombreux pays bilingues et multilingues, en Europe et ailleurs dans le monde (Mattila 2012). Mais quelle que soit la méthode que l'on suive ou prône, traduire (en général) n'en reste pas moins une «tâche» (au sens de Benjamin) complexe et délicate, en droit particulièrement, ne serait-ce qu'en raison de l'écueil que présente l'interprétation d'un texte de droit. Néanmoins, le but à atteindre demeure toujours la mythique «équivalence». Peut-on y parvenir quand on traduit le droit, domaine où prime le fait culturel, donc local?

Le Canada, qui est un des pays où la traduction, ce «mal nécessaire» (Gouin 1977: 31; Loertscher 2001: 81), joue depuis plusieurs siècles un rôle déterminant dans le fonctionnement de ses institutions, possède dans ce domaine une longue expérience, riche et pionnière. Plusieurs États partagent avec le Canada une situation de bilinguisme ou de multilinguisme, voire de bijuridisme ou de multisystémisme juridique. Tous connaissent, à un certain degré, des difficultés pour produire des lois bilingues ou multilingues. La jurilinguistique (Gémar et Kasirer 2005) est une des voies et un des moyens permettant d'y procéder. Née au Canada au début des années 1970, elle découle, à l'origine, de la traduction. Elle s'en est progressivement détachée pour s'appliquer à d'autres situations textuelles, en contexte unilingue par exemple, comme au Québec. Selon Covacs (1982: 86), «[p]ar un radical renversement de perspective, l'accent est d'entrée de jeu mis, non plus sur la traduction, mais sur la rédaction».

Ce saut qualitatif résume les progrès étonnants réalisés en l'espace de quelques générations, dus à la vision avant-gardiste des personnes et des institutions qui œuvrent à la préparation des lois, depuis leur conception et leur traitement jusqu'à leur mise au service d'un État bilingue, bijuridique et multiculturel. L'apport récent du monde judiciaire à ce chapitre est loin d'être quantité négligeable. La traduction en français des arrêts des juridictions canadiennes, notamment celle de la Cour suprême du Canada, a également contribué à l'amélioration du langage du droit exprimé en français. Ces progrès toutefois concernent «un État juridiquement, institutionnellement et linguistiquement complexe» (Cadiet 2004: 143), où le dialogue 
entre les langues et le droit est toujours fragile et incertain. Cause d'étonnement permanent, cette complexité, reconnue et déplorée à la fois par les juristes et par les linguistes, caractérise les États où règnent bilinguisme, bijuridisme et bisystémisme. La jurilinguistique, discipline que cette situation a engendrée au Canada, est une tentative de réponse aux difficultés que pose le traitement des textes juridiques en situation de bilinguisme et, facteur aggravant en l'occurrence, de bijuridisme bisystémique (Gaudreault 2012). Cette réponse, toutefois, doit être apportée non seulement sur le plan linguistique, mais - et cela complique d'autant plus les choses - également en accord avec les mœurs et traditions locales, soit sur un plan socioculturel (Gémar 2011: 129). Car la langue et ses usages se coulent difficilement dans une pensée conçue et exprimée dans un autre idiome. Pourtant, depuis la malédiction de Babel et afin d'en contrer les effets, l'humanité recourt à la traduction pour communiquer.

Lorsque l'on compare les situations d'un pays à l'autre, on peut se demander si le Canada est parvenu, après tant de vains efforts et de tâtonnements, à faire mieux connaître la loi, la rendre plus lisible et compréhensible à ses citoyens. Au terme de cette longue épopée, la traduction a-t-elle échoué au point de se voir supplantée par la corédaction? Et celle-ci est-elle la panacée, la solution miraculeuse que certains croient y trouver? Laquelle des deux permet-elle le mieux de réaliser cette "équivalence» dont on aimerait croire que le traducteur y parvient à tout coup au terme de son opération traduisante? Ne devrait-on pas plutôt en parler au pluriel: «(les) équivalences»? La sémantique, le foisonnement et la réalité des mots étant multiples, fugitifs et toujours interprétables, peut-on en dégager une valeur sûre, leur attribuer une Vérité unique? Finalement, l'interprétation du message juridique, traduit ou corédigé, en vue d'en fixer le sens ne serait-elle pas le seul critère valable du sens objectif à donner au texte de droit?

\section{La traduction, ce «mal nécessaire»}

La question: "Comment faut-il traduire?» se pose toujours, même si nombre de savants esprits (écrivains, philosophes, linguistes, traductologues, sociologues...) s'y sont essayés, s'efforçant d'apporter des réponses, des solutions, d'édifier un système cohérent, d'élaborer des principes et, parfois, des théories censés faciliter la «tâche» du traducteur. Néanmoins, tout traducteur le moindrement expérimenté sait que la traduction, art d'exécution, n'est pas une science exacte et qu'un texte ne se traduit pas à coups d'équations, de formules ou de grille qu'il suffirait d'appliquer pour obtenir automatiquement la réponse désirée - bonne, il va sans dire. Les difficultés que rencontrent les chercheurs en traduction automatique - notamment en sciences humaines et sociales - et les obstacles que la recherche en intelligence artificielle doit franchir en apportent continuellement la preuve: la machine à traduire est encore loin d'avoir égalé l'être pensant qu'est le traducteur. La traduction reste essentiellement humaine; les machines, soit les aides à la traduction, ne viennent qu'en appoint.

Aussi, s'agissant du droit et de la traduction de ses textes, importe-t-il de s'inspirer également des réflexions des juristes et, particulièrement, des comparatistes. Ces derniers sont en prise directe avec les problèmes que posent non seulement le langage et les mots du droit, mais aussi les notions et concepts qu'ils véhiculent d'un système à l'autre. Ceux-ci atteignent parfois un tel degré d'abstraction, de subtilité et de singularité que certains juristes comparatistes doutent de la 
possibilité d'une équivalence quand on passe d'un système juridique à un autre (David 1974; Legrand 2005).

Comme toute discipline fondée sur l'usage d'une langue, le droit présente son lot de difficultés et de problèmes, à commencer par le sens du terme fondateur luimême: droit, et l'interprétation qui peut en être faite selon le lieu et l'époque. Les juristes sont d'ailleurs les premiers à relever et même à dénoncer ces ambiguïtés, lacunes et faiblesses ${ }^{1}$. Lorsqu'il est réalisé en discours (Darbelnet 1982), le langage du droit s'inscrit dans un texte. La typologie des textes juridiques n'est pas sans intérêt et peut guider le traducteur dans ses choix de stratégie.

On connaît la distinction classique que font les juristes entre les catégories principales de textes qu'ils produisent: loi, acte, jugement, doctrine. Les traductologues ne sont pas en reste qui distinguent texte «document» et texte «instrument» et, en poussant plus loin la réflexion, envisagent, comme Sabatini (Megale 2008: 84) et Reiss (Megale 2008: 130), une typologie tripartite. Pour Sabatini, les textes se subdivisent en trois catégories fondamentales de textes contraignants: du plus au moins contraignant, en passant par le moyennement contraignant. La première comprend trois sous-catégories (textes scientifiques, normatifs, technico-opératifs), la deuxième, deux (textes explicatifs-argumentatifs, informatifs) et la troisième, une seule (les textes littéraires/esthétiques). Le texte juridique porteur de règles, tels la loi, le jugement et le contrat, entre manifestement dans la sous-catégorie des textes normatifs.

Il n'en faut pas moins se garder de voir dans le langage du droit une unité, qui n'est que de façade. À cet égard, il faudrait plutôt parler des langages du droit, selon que l'auteur du texte est le législateur, le juge, l'homme de loi ou le notaire, outre les subdivisions que l'on peut faire dans chacune de ces catégories de texte. À chaque grande fonction (exécutif, législatif, judiciaire) que la langue de Thémis doit exprimer correspondent un style et une manière de dire, une phraséologie particulière. Aussi ces catégories restent-elles le plus souvent abstraites, un texte pouvant réunir plusieurs fonctions (un jugement, par exemple, peut être à la fois normatif, informatif, technico-opératif, argumentatif, ainsi de suite), voire, en traduction, assurer des fonctions différentes entre le texte de départ et celui d'arrivée, comme certains auteurs de la Skopostheorie l'ont montré (voir Nord 2010: 183), même s'ils n'ont fait que mettre en évidence ce que tout traducteur (juridique) sait d'expérience.

Le problème que posent ces textes lorsqu'il s'agit de les traduire ne se résume pas à un vocabulaire, aux termes et aux notions (juridiques) qu'ils véhiculent. La façon dont est rédigé un texte juridique ne doit pas être sous-estimée. Le style des textes juridiques varie d'une langue à l'autre, parfois de façon considérable. Tel est le cas en particulier entre l'anglais et le français. Mais il varie aussi au sein du droit lui-même: style des lois, des jugements, des contrats, des traités, ainsi de suite. Ce style est celui que les grands juristes ont imprimé au cours de l'histoire du droit d'un pays, qu'ils ont illustré dans leurs écrits, doctrinaux ou jurisprudentiels. L'anglais juxtapose, place souvent les conditions en tête de phrase, d'article, de disposition ou de clause. Alors qu'en français, on pose un principe général, sous-entendant des choses censément connues. "À qui sait comprendre, peu de mots suffisent», disait Stendhal. Le verbe, mot porteur d'une charge sémantique maximale, est souvent placé en tête, comme ici:

Sont immeubles les fonds de terre, les constructions et ouvrages... (art. 900 C.civ.)

Font partie intégrante d'un immeuble les meubles qui... (art. 901 C.civ.) 
Deux styles, deux esprits. Pour reprendre le brillant raccourci de l'essayiste Michael Edwards, l'anglais est ancré dans le réel, alors que le français se place «dans un monde à la fois réel et cérébral »; la syntaxe anglaise oblige le rédacteur «à passer d'un événement à l'autre, alors que la syntaxe française plane un peu au-dessus de l'événement (...) et le dit avec un début, un milieu et une fin " ${ }^{2}$. S’il fallait les qualifier d'un mot, on pourrait dire, avec Edwards, que l'anglais est "centrifuge", alors que le français serait «centripète». On voit par là la difficulté que pose la reformulation du texte juridique d'une langue dans une autre, obstacle que tant de traducteurs réussissent pourtant à franchir quotidiennement, quelles que soient la longueur et les circonvolutions des phrases.

\section{Traduire le langage du droit : enjeux et méthodes}

Traduire est réputé difficile, mais traduire des textes juridiques l'est plus encore. Selon l'éminent juriste Gérard Cornu, «là où ils s'additionnent, le bilinguisme et le bijuridisme portent au paroxysme la complexité»(Snow 1995: 13). Il s'ensuit que la traduction juridique, tout particulièrement entre le français et l'anglais, pose un problème particulièrement aigu au traducteur. Il lui faut non seulement passer d'une langue à une autre, d'un système à un autre, mais encore d'une famille juridique à une autre. Rien de plus banal en soi, c'est le lot de la traduction juridique. Mais dans le cas de la common law face au système civiliste (qui appartient à la grande famille romano-germanique), nous avons affaire à deux systèmes, deux familles de droit fort différents l'un de l'autre parce qu'ils fonctionnent à partir de schémas de pensée et de méthodes opposés, situés aux deux extrémités du spectre et de la pensée juridiques: induction vs déduction, particulier vs général, jurisprudence vs loi, droit non écrit/coutumier vs droit codifié/écrit. Comme l'a si bien résumé René David, le grand comparatiste, en common law «la procédure prime le droit» (remedies precede rights), alors que dans la tradition civiliste, c'est le contraire qui prévaut.

Aussi, avant de songer à traduire et de rechercher des équivalents entre les langues, doit-on se demander comment réaliser l'équivalence.

\subsection{La quête d'une équivalence}

La plupart des pays ont recouru et recourent encore tantôt à la traduction littérale, tantôt à la traduction libre, mais également aux différentes manières de combiner l'une et l'autre. Chaque méthode possède ses partisans et ses adversaires. Le traducteur peut avoir à choisir entre les multiples possibilités et combinaisons distinguant ou rapprochant l'une de l'autre. Des querelles doctrinales divisent fréquemment les traductologues, en droit comme dans d'autres disciplines. Depuis que l'on traduit, soit depuis toujours ou presque (Horguelin 1981), on s'interroge sur la façon de produire un texte qui reflète au mieux le message du texte de départ. Au fil du temps, tous les grands traducteurs, parallèlement aux linguistes, se sont un jour ou l'autre posé la même question. Chacun a apporté des réponses et ses réflexions, souvent originales, sur la plupart des grands problèmes que pose la traduction et que des traductologues (p. ex., Ballard 1995; Horguelin 1981; Vinay 1981) n'ont pas manqué de relever, entre autres: la fidélité, la transparence, la modulation, la surtraduction, 
les variantes stylistiques, la stylistique interne, les lacunes, les niveaux stylistiques, l'adaptation et les limites de la traduction (voir Horguelin 1981 : 11).

Les juristes ne sont pas en reste. Ils ont, eux aussi, dans les traces des grands anciens (de Cicéron à nos jours), réfléchi, trouvé des réponses et proposé des solutions aux problèmes et difficultés qu'ils rencontrent lorsqu'ils ont à traduire des textes juridiques et à appliquer les principes de «l'art de traduire» (d'Alembert 1763). Si l'on ne devait prendre en compte que les sources d'inspiration des théories contemporaines de la traduction au $\mathrm{xx}^{\mathrm{e}}$ siècle, il faudrait partir de la linguistique saussurienne, passer par les speech acts d'Austin et Searle, la théorie de Sapir-Whorf, le structuralisme, la linguistique du texte et tant d'autres principes et éléments théoriques que l'on peut appliquer aussi au langage du droit, et on finira par les derniers développements de la traductologie, perçue comme transdiscipline. On y traverse de nombreux courants - dont celui de Chomsky, très influent parmi les traductologues - et on y croise de nombreux auteurs, depuis Jakobson, Nida, Holmes et Toury, jusqu'à Derrida et son principe de «déconstruction» qui envisage la traduction comme opérateur de «différance». On conclura provisoirement cette liste par la théorie du skopos, avec ses sources et ses nombreux avatars, dont celui de l'éthique selon Christiane Nord (2004: 234), avec son principe de loyauté, dans lequel on peut voir une version plus équilibrée de cette théorie. Le tout concourt à établir un cadre fonctionnaliste propre à répondre de façon optimale aux besoins de la traduction juridique.

Aussi le point de vue des juristes sur l'art et la manière de traduire est-il intéressant à plusieurs titres, car le texte juridique présente des difficultés particulières pour le traducteur, comme nombre de juristes l'ont souligné (voir David 1974: 346; Beaupré 1987: 735; Sacco 1999: 163; Legrand 2005). L’une des plus fréquentes vient de la présence, dans le texte à traduire, de termes portant une notion étrangère à la langue cible, auxquels le traducteur doit trouver l'équivalent dans le texte d'arrivée. Dans le cas moins sensible d'un texte autre que le juridique, le néologisme, le calque, voire l'emprunt, peuvent fournir des solutions acceptables selon les points de vue, le contexte et la destination du texte, sa «localisation». Ce type de solution toutefois ne se prête pas à toutes les situations (traité de droit, loi, jugement, contrat, traité, etc.). Le regard averti que jettent les juristes comparatistes sur ces difficultés, tant notionnelles que terminologiques, est éclairant pour le traducteur, car traduire des textes de nature ou de portée juridique revient à accomplir un acte de droit comparé, mais couplé à une opération traduisante. Telle est, en somme, la tâche redoutable du traducteur, qu'il soit juriste ou non.

Or, juristes et traducteurs ont beaucoup en commun. Tout d'abord, le comparatiste et le traducteur "interprètent» des textes, quoique à des fins et selon des méthodes différentes. Pour un juriste comparatiste comme Rodolfo Sacco, figure de proue du droit comparé contemporain, l'interprétation des termes linguistiques exprimant des concepts juridiques est un des problèmes majeurs du droit comparé (voir Sacco 1999: 168). Voilà qui est de nature à soulager le traducteur, qui, partant de la signification des termes (et des mots) du texte, doit arriver, à l'issue de son «interprétation», à dégager le sens du message avant de le réexprimer dans son texte d'arrivée. 


\subsection{L'équivalence: une cause commune?}

Si l'on s'en tient aux notions que recouvrent les termes principaux des vocabulaires du droit des principaux systèmes et que l'on en fait l'analyse comparée terme à terme, on aboutit la plupart du temps, faute d'équivalence parfaite, à l'impossibilité de traduire. On sait que le contract de la common law n'est pas le contrat du droit français; les biens meubles du Code civil ne sont pas l'équivalent de l'allemand bewegliche Sachen (terme dont la notion, contrairement au français, ne porte que sur des objets corporels). Certes. Mais, nous dit Sacco, la règle est la même, ce sont les concepts qui diffèrent, et l'interprétation «est faite sur la base de la façon dont l'interprète évalue les possibles solutions, et non sur la base du lexique» (Sacco 2002: 238). En outre, quand il s'agit de traduire le droit, il est vain de chercher une équivalence parfaite. L'enveloppe linguistique du concept est somme toute secondaire; ce qui compte, c'est non pas l'équivalence des concepts, mais celle des textes. Voilà le but à atteindre. C'est une affaire de degrés, mais aussi une question d'ordre pragmatique puisque l'équivalence peut être (juridiquement) complète, plus ou moins partielle, voire carrément impossible à réaliser: "On voit pourquoi il est tout simplement impossible de traduire en français avec une précision parfaite les termes techniques du vocabulaire de la common law" (Pigeon 1982: 275).

Ce n'est pas tant le terme et sa notion qui posent un problème au traducteur que la portée, les effets juridiques de «l'équivalent»: sont-ils ou non les mêmes que ceux du texte de départ? D’où les enjeux, réels ou supposés, de l'équivalence. Ils sont à la source de la réflexion des juristes sur la meilleure façon de la réaliser. Dès lors, s'est imposée l'idée que rédiger, et non plus traduire, la loi en parallèle dans chacune des langues officielles permettrait de produire un texte idiomatique et «lisible».

\section{L'équivalence et ses mythes: une «tétralogie»?}

Dans cette quête d'une équivalence, c'est celle de «l'esprit» des lois plus que de leur lettre que l'on cherche à rendre. Au Canada, « [1] a traduction par équivalence est [...] le procédé dominant» (Pigeon 1982: 276). C’est le cas de la plupart des pays et des personnes qui recourent à la traduction, juridique ou non. Le terme «équivalence» (des mots; des textes: TD = TA) vient de loin. Ses origines se perdent dans l'histoire de la traduction, mais ce n'est qu'au XIX ${ }^{\mathrm{e}}$ siècle (1864, selon le Robert historique) qu'il prend le sens qu'on lui connaît en linguistique. Vinay et Darbelnet (1958) le reprennent parmi leurs sept «procédés» de traduction, ce qui lui confère un statut quasi scientifique. Depuis, ce procédé de traduction, assimilé à une méthode, n’a cessé de se répandre dans les milieux de la traductologie. Il est généralement assorti d'un qualificatif, qui diffère selon les écoles de pensée et leurs théoriciens: (équivalence) connotative, dynamique, fonctionnelle, formelle, naturelle, référentielle, sémantique, textuelle, ainsi de suite.

En droit, le principe de l'équivalence paraît relativement simple: on assume que, quel que soit le système juridique, des problèmes identiques se présentent partout qui appellent des solutions identiques. Ces problèmes sont toutefois résolus par des moyens différents, dont une procédure judiciaire singulière. L'ennui, nous dit le comparatiste Olivier Moréteau, «est que l'on ne trouve pas toujours une institution ou une technique équivalente» (Megale 2008: 99). Aussi l'équivalence dite "fonc- 
tionnelle» passe-t-elle, en droit comparé, pour la solution privilégiée en présence de systèmes comparables. Si l'équivalence fonctionnelle n'est pas la solution idéale (si tant est qu'il y en ait une), même entre systèmes proches ${ }^{3}$, elle n'en constitue pas moins un "pis-aller», un "accommodement raisonnable» que suivent les comparatistes depuis des décennies et qui, en l'absence d'une terminologie neutre ou d'une utopique ontologie, est généralement préférable aux six autres procédés de traduction (calque, emprunt, traduction littérale, oblique, etc.), auxquels il est toujours possible de recourir selon les situations.

Appliqué à la traduction de textes juridiques, le principe de l'équivalence a été associé au fonctionnalisme, qui semble tenir la corde avec le terme "équivalence fonctionnelle», auquel le juge Pigeon a donné sa caution. Toutefois, lorsque l'on évoque le terme équivalence, qui nous vient du vocabulaire de la science, on ne mesure pas clairement ce que réserve son application à la situation textuelle particulière que présente la traduction de tel texte juridique, qu'il s'agisse d'un «document» ou d'un «instrument». Or, ces situations sont potentiellement innombrables. Elles découlent des circonstances et des conditions dans lesquelles le texte (de départ, en traduction) a été produit et des objectifs (dont la ou les fonctions dudit texte) que visaient ses auteurs. Le texte, toujours recommencé, est néanmoins unique. Le message qu'il porte repose sur le principe, le concept ou la notion juridique que véhicule le langage du droit, dont le fondement est constitué des termes du vocabulaire juridique. Selon le regard que l'on portera sur ce vocabulaire (par exemple, celui d'un historien, d'un comparatiste, d'un philologue, d'un philosophe, d'un juriste, d'un sociologue, d'un comparatiste...), sa typologie variera de façon importante. Selon les buts visés, il est possible de découper le vocabulaire juridique en plusieurs catégories, par exemple en vue d'élaborer un dictionnaire, un vocabulaire ou un lexique. Aux fins de la traduction, ces termes peuvent être classés en trois catégories principales, comme l'a fait Jean Kerby:

Dans la traduction juridique de l'anglais au français, on trouve trois sortes de termes: ceux qui ont un équivalent sémantique français (offer et offre); ceux qui n'ont pas d'équivalent exact en français mais pour lesquels on peut trouver un équivalent fonctionnel (mortgage et hypothèque) et ceux qui sont carrément intraduisibles (common law, equity...). (Pigeon 1982: 277)

Je reprends cette classification tripartite, classique à cette époque-là (1979), en la précisant et la développant quelque peu. C'est ainsi que je lui ajoute une introduction, acte préalable qui, suivi des trois autres, en fait une «tétralogie de l'équivalence», pour emprunter cette métaphore musicale à Wagner. Je mappuie pour cela sur la réalité du phénomène de la "dérivation» linguistique: la variabilité des termes et notions est incontournable. La valeur de la plupart des termes juridiques, en effet, est susceptible de varier dans le temps, sur le long comme sur le court terme. Certains termes toutefois, notions incluses, sont (relativement) «invariants». Il s'ensuit parfois un décalage entre la notion de départ et celle d'arrivée, alors qu'elles pouvaient être considérées jusque-là comme étant (relativement) équivalentes. Ce n'est qu'une des nombreuses difficultés que pose l'opération traduisante au traducteur. 


\subsection{La mise en scène de l'équivalence}

Avant de se lancer dans l'opération traduisante, il importe que le traducteur effectue un parcours s'apparentant à la visite des «tableaux d'une exposition » (Moussorgski), pour continuer à filer la métaphore musicale. Chaque terme, comme chaque tableau, est un concentré d'histoire, celle d'une notion, d'une institution ou d'un concept juridiques, que le traducteur doit retracer et parcourir, en diachronie, afin d'en saisir le sens dans toutes ses nuances et d'en comprendre la portée avant de les reproduire, en synchronie, dans son texte d'arrivée. Muni de cette information, il passera plus aisément à l'étape suivante de la comparaison de ces données avec celles du terme potentiellement équivalent dans l'autre langue et dans l'autre système.

La principale difficulté réside dans la notion que porte le terme. Un exemple en fera prendre la mesure, celui du terme anglais property. Si on le compare avec son prétendu équivalent bien (ou propriété, selon son autre sens), à première vue, les notions de bien et de propriété clairement définies en droit civiliste ne soulèvent pas de grandes difficultés au sein de ce système juridique. Mais si l'on sort du cadre d'un monosystémisme et d'un unilinguisme juridiques (au Brésil, en Russie, aux ÉtatsUnis ou en France, par ex.) et que l'on cherche à comparer termes et notions et leurs équivalents dans une langue et un système étrangers, celui de la common law par exemple, la complexité de l'opération traduisante apparaît d'emblée.

En common law, contrairement au droit civiliste, la notion de bien (ou de propriété) n'a pas de définition précise. En outre, les mots sont impuissants à décrire la totalité des realia lorsque, en anglais, property peut aussi bien signifier (droit de) propriété (parfois) que bien (plus souvent). Et la difficulté s'accroît quand on sait que les analyses diffèrent d'un système à l'autre lorsqu'il s'agit de comparer les notions de bien/property et de propriétélownership. La situation particulière du Québec dans le vaste domaine du droit comparé autorise la rencontre - ou la jonction? - d'un droit féodal marqué par ses origines coutumières (la common law, droit "non écrit»), et d'un droit savant - écrit, celui-ci - de tradition civiliste. Le langage du droit et ses notions fondamentales s'en ressentent dans ce qu'ils possèdent de plus fondamental: la propriété (ownership/property). La comparaison s'arrête à la lisière des termes puisque, en common law, seule la Couronne (the Crown) est propriétaire de la terre, la part de l'individu étant réduite à des intérêts, alors qu'en droit civil, c'est ce dernier qui, sauf exception (démembrement, servitudes, etc.), est le plein propriétaire d'un bien-fonds en vertu de l'abusus, attribut essentiel du droit de propriété.

Mais que dire de la comparaison des notions de trust et de fiducie (son équivalent, au Canada) entre la common law et le droit civiliste? L’idée que la propriété puisse être multiple et divisible est, pour un juriste français, inconcevable au regard du caractère unique et indivisible de la propriété.

Ces quelques exemples montrent l'importance de l'analyse comparative minimale à laquelle devrait procéder toute personne désirant traduire un texte juridique, quels que soient les systèmes de droit et les langues en cause.

Ces termes peuvent être classés en trois groupes principaux, au moins. Dans le premier, je place les termes dont l'équivalence est évidente, reconnue ou établie; dans le deuxième vont les termes pour lesquels l'équivalence n'est que (plus ou moins) partielle et, dans le troisième, ceux des termes dont la traduction est impossible pour diverses raisons (nom propre; absence de correspondance de la notion/institution 
dans un autre système; etc.). Les deux premiers groupes entrent dans la définition de ce qu'il est convenu d'appeler «l'équivalence fonctionnelle ${ }^{4} »$. Le troisième correspond dans la plupart des cas à ce qu'il est convenu d'appeler un «emprunt» ou un «calque», ce qui équivaut à un renoncement de la part de son auteur, incapable de trouver un terme équivalent dans sa langue. Ici, la langue prime le langage et nous rappelle la malédiction de Babel.

\subsection{L'équivalence: une concordance par défaut}

Lorsque ce langage singulier qu'est celui du droit doit être transposé dans une autre langue, la difficulté linguistique s'ajoute à la complexité juridique. La linguistique nous rappelle ce principe incontournable: la notion (juridique, en l'espèce) et l'image mentale associées à un signe linguistique, parce qu'elles sont propres à une langue et au terme qui les véhiculent, passent mal d'un système de signes à un autre. Et quand cela se fait, en traduction et en interprétation, c'est au prix d'un compromis juridicolinguistique fonctionnel ou «raisonnable». C'est la démonstration implacable qu'en font Vinay et Darbelnet (voir 1958: 58) et Georges Mounin (voir 1953: 55), chacun avec ses arguments linguistiques, les premiers opposant le plan du réel à celui de l'entendement, et le second l'action à sa modalité.

En traduction spécialisée, contrairement à la traduction littéraire - où c'est le style, l'agencement des mots par l'auteur, qui fait l'œuvre -, ce sont les termes auxquels il faut trouver un équivalent, parce qu'ils sont porteurs de la notion et du message techniques. En droit, ce principe est fondamental et incontournable: on ne peut se satisfaire d'ignorer le terme de départ dans la traduction d'une loi, d'un jugement, d'un contrat, au risque de la voir déclarée fautive ou insatisfaisante.

Un exemple devrait suffire à illustrer ces différences, que j'ai souvent utilisé, celui de rule of law, et de ce que l'on tient pour son équivalent français: État de droit (aussi: primauté $d u$ droit, selon le contexte). Une analyse sociopolitique minimale ferait ressortir la différence d'approche entre les deux langues et leurs systèmes: la partie vs le tout, le particulier vs le général, ainsi de suite. On pourrait en conclure que la traduction terme à terme s'avère, ici, impossible sans recourir à une paraphrase pour expliquer le fondement de chacun des termes et le faire ainsi comprendre à l'autre communauté linguistique et, le cas échéant, juridique. Or, il se trouve que chaque grande démocratie évoluée - dont le Royaume-Uni, le Canada, la France, l’Allemagne, les États-Unis, à titre d'exemple - présente plus ou moins les mêmes caractéristiques, lorsqu'il s'agit de désigner un concept (par ex., la démocratie), une institution (par ex., le contrat) ou un principe (par ex., la présomption d'innocence), soit des situations communes que chacun partage avec les autres. Ce qui est le cas de la "primauté du droit». Il suffit alors de prendre le terme qui, dans chaque État, désigne ce concept commun: Rechtsstaat (allemand), estado de Derecho (espagnol), stato di diritto (italien), entre autres. L'équivalence "fonctionnelle», dans ce cas, n'est rien d'autre qu'une concordance, toute relative, des fondements d'une notion, d'une institution, d'un principe, que tous partagent. Les modalités d'application, elles, diffèrent dans chaque cas, chacun des États considérés possédant ses propres règles et sa procédure. Un terme comme obiter (dictum) se rend, au Canada, par obiter, tout simplement. Le terme constitution résume à lui seul la difficulté du genre; celle du Royaume-Uni, de tradition non écrite, a peu à voir avec celles de la France, de l'Espagne ou des 
États-Unis, qui sont écrites. Dans un tel cas, l'équivalent français (ou allemand, espagnol, etc.) constitution ne peut être que «fonctionnel».

On pourrait allonger indéfiniment, et inutilement ici, la liste des équivalences fonctionnelles, dont les exemples pullulent. À propos d'un terme comme property, il suffira de dire, en français, bien mobilier ou immobilier selon le cas. Dans le cas de tort, s'il fallait désambiguïser ce terme, on ajouterait à son équivalent naturel délit l'adjectif civil (délit civil). Un dernier exemple: mortgage (hypothèque), qui illustre la variation notionnelle. Dans l'univers de la common law (Québec inclus), l'hypothèque peut être soit mobilière, soit immobilière ${ }^{5}$, ce qui va à l'encontre de la tradition civiliste, pour laquelle l'hypothèque est une "sûreté réelle immobilière» (Cornu). Là encore, il suffira de préciser, dans le texte français, hypothèque «mobilière» ou «immobilière», selon le cas. Dans tous ces cas, au vu des écarts notionnels, l'équivalence peut alors être qualifiée de «fonctionnelle». Il y a toutefois de nombreux cas et situations où, pour nombre de raisons, la concordance des termes, sans être irréductible, n'est que partielle, ce qui pose un tout autre problème d'équivalence au traducteur.

\subsection{L'équivalence partielle: le plus et le moins-disant}

C'est sans doute le cas le plus fréquent auquel le traducteur juridique (tout traducteur, en fait) doit faire face lorsque les notions portées par le terme à traduire ne concordent pas exactement avec l'équivalent considéré. Là encore, les exemples abondent entre la common law et le système civiliste. En voici quelques-uns: contract et contrat; trust et fiducie (en France); crime et crime; burglary et vol avec effraction ${ }^{6}$. On pourrait pousser la réflexion jusqu'à envisager des niveaux de concordance et des degrés (+ ou -) d'équivalence, dans une grille ou selon un procédé propre à mesurer l'écart notionnel séparant les termes en deux catégories, soit entre ceux qui «disent le moins» et ceux qui «disent le plus», par rapport à la notion et au terme d'arrivée. On pourrait reprendre ici l'idée du plus grand et du plus petit commun dénominateur notionnel ou sémantique que j'ai présentée ailleurs (voir Gémar 2007: 196). Faut-il aller jusque-là?

Arrêtons-nous à l'exemple du contract de la common law pour illustrer mon propos. Pour le profane, les deux termes, contract et son pendant français contrat paraissent équivalents. Le comparatiste, lui, sait bien qu'il n'en est rien. Si la plupart des conditions essentielles de validité du contrat sont identiques ou voisines dans les deux systèmes (capacité de contracter, offre et acceptation, etc.), en revanche, la condition de consideration de la common law, qui est essentielle ( the contract»), n'existe pas en français. Il faut alors introduire, dans la version française, une "béquille» pour rétablir l'équilibre: «moyennant/en contrepartie de/la somme de...», faute de quoi les deux versions ne seraient pas jugées fonctionnellement équivalentes, avec les conséquences juridiques que l'on imagine.

Si la différence avec le groupe précédent est mince, doit-on reconnaître, elle n'en existe pas moins. Dans le premier groupe, l'écart est d'ordre conceptuel', dans le second, de nature notionnelle ${ }^{8}$. Ce qui nous conduit au quatrième et dernier acte de cette saga où le sort «crépusculaire» de l'équivalence des termes est mis en évidence. 


\subsection{Traduire ou ne pas traduire le terme? Voilà la question...}

Dans toutes les langues, il existe des mots ou termes, en nombre variable selon les familles linguistiques, désignant une réalité propre à un groupe linguistique, une culture, et qui n'ont pas d'équivalent(s) dans les autres langues. On connaît le cas de l'inuktitut et de ses nombreux vocables pour qualifier la neige sous tous ses aspects; de même que celui de l'espagnol des «gauchos» argentins pour qualifier la couleur de la robe de leurs chevaux. Ce phénomène est universel et touche tous les secteurs de l'activité humaine, dont le droit et son langage. Les deux systèmes de droit en cause, la common law et le système civiliste, n'en sont pas exempts, même si le nombre de ces termes n'est pas aussi élevé que l'on pourrait croire.

Dans ces cas-là, par exemple ceux de termes ou noms propres invariants tels que common law, equity, il n'existe pas d'équivalent, naturel ou autre, dans les autres langues. La traduction est ici véritablement impossible et conduirait, sinon, à des solutions absurdes, comme le soulignent des spécialistes du droit parmi les plus éminents, juristes et juges confondus (R. David, L.-P. Pigeon, R. Sacco, etc.), à propos, entre autres, de ces deux termes. En effet, droit commun comme traduction de common law et équité pour rendre l'anglais equity seraient des faux sens graves qui dénatureraient le sens du concept originel.

Il existe de nombreux autres termes, moins voyants pour la plupart, désignant une institution, un droit ou un acte de procédure. Sans être intraduisibles pour autant, ils posent, chacun dans son genre et domaine, des difficultés au traducteur pour en rendre toute la charge sémantique. Par exemple: chattel = bien meuble, alors que, d'après l'étymologie, on s'attendrait à une chose "immobilière» (un «bienfonds»)! [Voir chattel mortgage: hypothèque mobilière] ; corporation (cinq significations différentes!); damage(s) [ce $s$ fait toute la différence...]; due process (of law); trespass; trust, vu plus haut; et ainsi de suite.

Cette «tétralogie» de l'équivalence n'épuise cependant pas le sujet, car elle ne porte que sur des mots, termes et expressions, même s'ils constituent le fondement du langage du droit. Pour ma part, l'équivalence passe aussi par le discours, l'agencement des mots, soit le style de rédaction propre à chaque tradition juridique et, en son sein, à chaque genre de texte porteur de règles de droit (Gémar 2007). Je prétends que ce style est porteur de sens en ce qu'il correspond à une culture juridique propre. Chaque État bilingue ou multilingue, chaque organisation internationale a forgé sa propre méthode de production de ses textes juridiques dans d'autres langues, notamment de ses textes législatifs. Certains, la majorité, les traduisent; d'autres, minoritaires encore, préfèrent les rédiger. D’autres enfin, recourent tantôt à la rédaction pour certains de leurs textes (l'Union européenne, par ex.) afin de mieux en garantir l'égalité linguistique (parfois décrétée par le législateur, comme en Belgique), et tantôt à la traduction des autres textes (l'UE, encore).

On peut alors s'interroger sur les mérites de l'un (la traduction) et de l'autre (la corédaction) modes d'expression des lois bilingues. La traduction (des lois) a-t-elle démérité au point d'être supplantée par la corédaction? La réponse se trouve peutêtre en partie dans l'interprétation des lois lorsque l'interprète de la loi juge que les deux versions sont ou non équivalentes, ce qui déterminera si en elles font ou non également foi. 


\section{Corédiger les lois comme méthode de «traduction"}

Le Canada est un État fédéral à la tête d'un territoire immense, qui occupe le deuxième dans le monde pour ce qui est de la superficie. Pour cette raison, notamment, il lui a fallu trouver et mettre rapidement en œuvre des modes de gouvernance adaptés à des contraintes géographiques que des États comme la Belgique, la Suisse ou la Finlande ne connaissent pas au même degré. C'est ainsi qu'il a procédé à une centralisation de certains de ses services et créé, en 1934, un Bureau de la traduction qui regroupe tous les services de traduction des ministères du gouvernement fédéral. Il en sera de même pour la production de ses lois. Dès la fin des années 1940, le regroupement des services de rédaction législative du gouvernement fédéral s'effectue progressivement au ministère de la Justice du Canada. La Direction des services législatifs sera chargée d'étudier et de rédiger les projets de loi et les amendements.

Quelque 60 ans plus tard, on peut tenter d'esquisser un bilan de cette action, dont la corédaction n'est pas la moindre des curiosités et, finalement, des réussites? Elle constitue une source d'inspiration pour de nombreux pays, ne serait-ce que parce qu'elle porte sur les deux grandes langues véhiculaires et les deux principaux systèmes et cultures juridiques du monde occidental. De plus, l'expérience canadienne de la traduction montre que l'on peut trouver des solutions à certains des problèmes les plus aigus posés par les textes juridiques, anglais ou français, lorsqu'il s'agit de les traduire. Il est possible d'introduire une plus grande simplicité, clarté et concision dans l'expression du droit que celles que présente le texte de départ anglais (voir Mattila 2006: 232). Les lois corédigées le démontrent amplement, mais la traduction, entre autres celle des contrats, tout autant. Laccent est davantage mis sur la «lisibilité» du texte et les préoccupations stylistiques se sont accentuées. La complexité du droit et de ses notions n'en demeure pas moins un obstacle à la compréhension pour le commun des mortels. Il est désormais admis que «la traduction juridique doit être idiomatique, et non pas strictement littérale» (Flückiger 2005: 356).

Cette méthode (voir Labelle 2000: 269), qui a fait la part belle aux deux langues officielles, l'anglais et le français, en dépit de sa réussite, n'exclut pas les difficultés, notamment celles que pose l'interprétation des textes ${ }^{10}$, en particulier lorsque l'interprète de la loi fait passer ses choix culturels avant les options linguistiques du législateur. Quand la culture, la langue et le droit s'affrontent... faut-il souligner l'importance de la culture, en droit comme en traduction? L'exemple de l'interprétation des législations plurilingues va plus loin encore, jusqu'à la disparition même, sur le plan matériel, du texte, qui est en réalité un métatexte constitué de toutes les versions linguistiques successives, ce qui a pour curieux effet, s'agissant de textes législatifs bilingues et bijuridiques (cas du Canada), d'orienter une démarche herméneutique vers des critères téléologiques fort étrangers à la méthode d'interprétation traditionnelle de ce droit - la common law, en l'occurrence. Bref, la corédaction de ces textes ne simplifie pas pour autant leur interprétation. Susan Šarčević (voir 2005: 283) donne un exemple montrant un des effets possibles de cette technique qui peut conduire à briser des règles «sacrées " en traduction juridique, mais tolérées en corédaction, quand l'article 10 (9) de la Loi sur la sûreté du transport maritime (1994, ch. 40) énonce ce qui suit: 
The Minister may revoke the approval of security rules, either at the request of the operator or otherwise.

[18 mots]
L’approbation est révocable.

[4 mots]

La disposition anglaise de dix-huit mots n'en fait que quatre dans la version française, jugée «équivalente». En l'occurrence, le droit fédéral canadien s'accommode des contraintes que peut imposer la rédaction idiomatique de ses lois. Mais qu'en est-il de l'interprétation des deux versions à laquelle une telle situation peut donner lieu?

\section{Interpréter la loi traduite ou corédigée}

Le sujet de l'interprétation des lois est trop important et trop vaste pour prétendre en faire le tour dans le cadre d'une courte partie d'article. Je me borne à en présenter ici quelques aspects d'intérêt pour la comparaison des deux techniques d'expression des lois, traduire/corédiger, dans le contexte évoqué ci-dessus.

Les sciences sociales, la traductologie et le droit ont en commun des théories, des principes et des méthodes d'interprétation de leurs textes. Elles partagent même, en apparence, une alternative fondamentale. D'un côté, le traducteur a le choix entre traduire de manière littérale ou libre. De l'autre, chez les juristes, l'interprétation d'une loi peut être stricte (ou restrictive) ou large: stricto sensu ou lato sensu.

La ressemblance toutefois s'arrête là, car, en pratique, les choses sont bien plus complexes qu'il n'y paraît, d'un côté comme de l'autre. Pour le traducteur, c'est le sens du «message» que porte le texte de départ ${ }^{11}$ - et non ses mots - qu'il faut «interpréter $»^{12}$ avant de le réexprimer dans un texte d'arrivée. Pour le juriste, et pour les juges en particulier, le sens du texte de loi à interpréter passe généralement par l'intention du législateur (ou du Parlement). Une fois acquis le principe de l'égale autorité des deux versions d'une loi, comme le prévoient la loi canadienne et celle d'autres États ou organisations, l'intention du législateur, qu'il faut établir, est l'obstacle à franchir. Selon une voix autorisée, celle d'un juge de la Cour suprême, c'est une démarche à haut risque (voir Bastarache 2005: 95). En effet, les deux systèmes de droit ne recourent pas aux mêmes techniques. Dans la tradition romano-germanique, pour établir l'intention du législateur, les travaux préparatoires tiennent une place importante pour éclairer la signification de l'acte (voir Cornu, 2003: 680). Dans la tradition de la common law, en revanche, on se méfie des débats parlementaires, source peu fiable de l'intention du législateur (voir Bastarache 2005: 105).

Si l'intention législative - qui est une fiction juridique destinée à pallier les lacunes que constitue, d'après H. L. A. Hart, «la texture infiniment variable de la langue» - est la «pierre angulaire» (Bastarache 2005: 95) de l'interprétation des lois, l'intention de l'auteur est le critère sur lequel se fonde le traducteur, herméneute du texte, pour «l'interpréter». La différence est tout entière dans la règle, impérative, qu'instaure le législateur. L'interprète de la loi «dit le droit», l'interprète du texte «dit le message» véhiculé. Mais c'est surtout l'interprétation des lois bilingues, davantage que celle des lois unilingues, qui retient l'attention du traducteur et du jurilinguiste. Or, contrairement à ce que l'on pourrait croire, la langue n'est pas toujours en cause; la version que retiendra le tribunal (la Cour suprême, en l'occurrence) est celle qui exprime le plus exactement ce qui semble correspondre au sens voulu (voir Bastarache 2005: 107). 
La Cour suprême, dans les cas de conflit entre les deux versions d'une loi, non seulement n'a pas retenu de préférence la version anglaise, mais elle s'est souvent prononcée en faveur de la version française, comme le montrent les arrêts de la Cour suprême (voir Bastarache 2005 : 114-116).

Il arrive parfois que la version rédigée dans l'autre langue soit plus claire, moins ambiguë, et que ce facteur détermine la décision du tribunal. Selon le juge Dickson, «lorsqu'une version de la Charte contient une ambiguïté, et que l'autre version est moins ambiguë, il faut alors retenir le sens de la version la moins ambiguë ${ }^{13} »$. On en conclura que «le caractère bilingue [du Canada] s'affirme de plus en plus », facilitant ainsi le rapprochement des deux systèmes juridiques, en sorte que «le recours aux deux versions d'une loi facilite le travail de la Cour», d'où l'intérêt d'adopter des lois bilingues (Bastarache 2005 : 116-117).

Le système de droit canadien accorde à la traduction (des lois) un statut d'égalité objective que la linguistique ne lui reconnaît que symboliquement par l'équivalence - toute relative - des textes. Ce que confirment la jurisprudence ${ }^{14}$ et la doctrine. Selon Popovici (2009: 229), «juridiquement la version anglaise d'un texte du Code civil du Québec a la même valeur que le texte français». Pour le traductologue toutefois, et cela dans tous les domaines, le sens porté par le texte de départ reste la référence absolue pour que le texte d'arrivée concorde avec celui de départ (Gémar 2006). Or, pour les tribunaux, l'important c'est «l'uniformité d'interprétation et d'application de la loi», alors que «son intelligibilité ne représente qu'un idéal» (Côté 2005: 143).

En somme, sur le seul plan de l'interprétation que les tribunaux peuvent faire d'un texte juridique, celle d'un texte corédigé comme celui de l'exemple donné plus haut (cf. Marine Transportation Security Act/Loi sur la sûreté du transport maritime) pourrait présenter quelques difficultés quant à «l'équivalence» des textes. Alors que des siècles d'interprétation par les tribunaux de textes traduits confèrent à la traduction une bonne longueur d'avance sur sa rivale, la corédaction. La traduction n'a pas encore dit son dernier mot.

Alors, existe-t-il une méthode de traduction garantissant l'équivalence totale à recommander? À l'évidence, non, et, en traduction juridique, encore moins. La meilleure théorie de la traduction n'a jamais aidé, non plus, un traducteur à produire un meilleur texte, car on ne traduit pas à coups de théories. Les principes théoriques avancés par les traductologues, aussi savants soient-ils, ne viennent qu'en appoint, parallèlement à une pratique qui a fait ses preuves. Si l'analyse des mécanismes qui entrent en jeu dans l'opération traduisante révèle néanmoins les subtilités et les difficultés de la démarche du traducteur, elle ne le guide pas dans son épreuve. Or, chaque nouveau texte à traduire est un nouveau défi, un cas d'espèce requérant une stratégie particulière du traducteur, dont le savoir-faire reste encore la meilleure garantie de succès dans son entreprise. On peut toujours élever le débat jusqu'à la philosophie, rendre ainsi le traducteur plus «intelligent», plus savant, ce qui est, somme toute, loin d'être négligeable.

Il n'est contraire ni à l'esprit ni à la syntaxe du français - ou d'autres langues - de dire une chose de la même manière, ou à peu près, que la langue de départ, quoiqu'il soit «toujours possible de dire la même chose autrement» (Ricœur 2004: 16). Ce qui pose la question de l'équivalence entre les deux textes. Il reste que la recherche d'un langage univoque, dénué d'ambiguïtés, est une préoccupation commune à tous les champs de l'activité humaine. Les juristes n'y échappent pas, pour lesquels le principe 
in claris cessat interpretatio peut laisser croire au mythe de la transparence et de l'univocité du langage que semblent créditer les divers mouvements de plain language et de «lisibilité» des textes juridiques.

\section{Conclusion : la traduction ou «l'imparfait du fugitif ${ }^{15}$ »}

Si «les mots sont bien la dernière chose sur quoi l'on parvient à s'entendre» (Droit 2007: 9), la comparaison des droits, comme la comparaison des mots et des textes, atteint vite ses limites. L'essence d'un système juridique est peut-être irréductible à toute transposition, donc à la traduction; d'où l'hypothèse de l'impossibilité de traduire le droit que posent certains juristes (Legrand 2005). L'arbre des mots, telle la pointe émergée de l'iceberg qui ne révèle qu'un fragment de sa masse gigantesque, cache la forêt des concepts, lesquels reposent sur un socle de plusieurs étages, catacombes où réside le mystère de leur origine. La plupart du temps, on croit saisir la vérité des mots alors que l'on ne survole que l'écume du sens, tapi dans les tréfonds du langage. Il reste que la traduction non seulement existe, mais prospère et se répand urbi et orbi. Dans le "village global» qu'est devenu le monde, la communication passe de plus en plus par elle, moyen incontournable de faire dialoguer l'humanité.

Dans le rapport étroit et continu qui lie la langue et le droit, la solution passera peut-être aussi par la jurilinguistique (Gémar et Kasirer 2005), passerelle jetée entre la langue et ses mots, d'une part, et le droit et ses textes, d'autre part, afin que la lettre et l'esprit des systèmes linguistique et juridique en présence cohabitent le plus harmonieusement possible. Il sera alors permis d'espérer que le traducteur arrivera à produire cette «traduzione giuridica alta» (la «haute» traduction juridique), que Sacco (Megale, 2008: 25) attribue au savoir du comparatiste.

\section{NOTES}

1. Voir Villey (1975: 222-226) et Le langage du droit, vol. XIX, Archives de philosophie du droit. Paris: Éditions Sirey.

2. http://bibliobs.nouvelobs.com/2008/08/14/a-wonderful-word-par-michael-edwards, consulté le 12 septembre 2012.

3. Voir les critiques que formulent Šarčević et de Groot dans MÉGALE, Fabrizio (2008: 100).

4. Qualification due à Pigeon, Louis-Philippe (1982: 271), juge à la Cour suprême du Canada.

5. «L'hypothèque est un droit réel sur un bien, meuble ou immeuble (...) OU [...]» (art. 2660 CCQ).

6. Voir, sur ce terme, le commentaire historique détaillé de Pigeon (1982: 273-276).

7. «Représentation mentale abstraite et générale, objective, stable, munie d'un support verbal» (Trésor de la langue française).www.lexilogos.com/francais_langue_dictionnaires.htm, consulté le 12 septembre 2012.

8. «Idée générale et abstraite en tant qu'elle implique les caractères essentiels de l'objet». Nous soulignons. (Trésor de la langue française) www.lexilogos.com/francais_langue_dictionnaires.htm, consulté le 12 septembre 2012.

9. Voir ŠARČEviĆ, Susan (2005: 277); en particulier: 1. A tribute to Canada, 279.

10. Sur cette délicate question d'interprétation des textes législatifs, voir Bastarache, Michel et al. (The Law of Bilingual Interpretation. Toronto: Butterworths, 2008) et sa version française : Le droit de l'interprétation bilingue. Montréal: LexisNexis, 2009.

11. Qu'il soit composé de quelques mots (par ex.: «Je pense, donc je suis») ou de centaines de pages (le Code civil, par ex.).

12. Au sens qu'Umberto Eco donne à ce mot, soit qu'interpréter est l'opération qui "précède toute traduction» (Eco 2006: 110), parce que «interpréter n'est pas traduire» (p. 265 et s.), mais est l'opération qui vise à élucider la signification du texte, sa «signifiance» (Barthes), en vue de le traduire. 
13. Mahé c. Alberta, [1990], 1 R.C.S. 342, par. 49.

14. Doré c. Verdun (Ville de), [1997] 2 R.C.S. 862, 150 D.L.R. (4e) 385.

15. Titre d'un ouvrage de Frédéric Musso (2010), poète, essayiste et journaliste, qui qualifie particulièrement bien la nature de la traduction.

\section{RÉFÉRENCES}

Alembert, Jean le Rond d' (1763): Observations sur l'art de traduire. Paris.

Ballard, Michel (1995): De Cicéron à Benjamin. Lille: Presses universitaires de Lille.

BASTARACHe, Michel (2005): Les difficultés relatives à la détermination de l'intention législative dans le contexte du bijuridisme et du bilinguisme législatif canadien. In: Jean-Claude GÉmar et Nicholas Kasirer, dir. Jurilinguistique: entre langues et droits. Jurilinguistics: Between Law and Language. Montréal/Bruxelles: Thémis/Bruylant, 93-117.

Beaupré, Michael (1987): La traduction juridique. Introduction. Cahiers de droit. 28:735-745.

CAdiEt, Loïs (2004) : Dictionnaire de la Justice. Paris: PUF

Coodec, George (1848): On Legislative Expression; or the Language of the Written Laws. Philadelphie: T. \& J. W. Johnson.

Cornu, Gérard (2003): Vocabulaire juridique. Quadrige. Paris: PUF.

CôTÉ, Pierre-André (2005): La tension entre l'intelligibilité et l'uniformité dans l'interprétation des lois plurilingues. In: Jean-Claude Gémar et Nicholas KAsırer, dir. La jurilinguistique: entre langues et droits. Montréal: Thémis, 123-143.

Covacs, Alexandre (1982): La réalisation de la version française des lois fédérales du Canada. In: Jean-Claude GÉmAR, dir. Langage du droit et traduction. Essais de jurilinguistique. Montréal/Québec: Linguatech/Conseil de la langue française, 83-100.

Crépeau, Paul A. (1993): L'affaire Daigle et la Cour suprême du Canada ou la méconnaissance de la tradition civiliste. In: Ernest CAPARros, dir. Mélanges Germain Brière. Montréal: Wilson \& Lafleur, 217-281.

DARBELNET, Jean (1982): Niveaux et réalisations du discours juridique. In: Jean-Claude GÉMAR, dir. Langage du droit et traduction. Essais de jurilinguistique. Montréal/Québec: Linguatech/ Conseil de la langue française, 51-60.

David, René (1974): Les grands systèmes de droit contemporains. $6^{e}$ éd. Paris: Dalloz.

Droit, Roger-Pol (2007): Éthique: envers les autres ou envers soi? Le Monde des livres. 2 mars 2007, 9.

Eco, Umberto (2006): Dire presque la même chose. Expériences de traduction. (Traduit par Myriem Bouzaher). Paris: Grasset.

FLÜCKIGER, Alexandre (2005): Le multilinguisme de l'Union européenne: un défi pour la qualité de la législation. In: Jean-Claude GÉmar et Nicholas KASIRER, dir. La jurilinguistique: entre langues et droits. Montréal: Thémis, 339-361.

Gaudreault, Marie-Claude (2012): Bijuridisme législatif: fondements et mode d'emploi. Bijuridisme et Harmonisation. Ministère de la Justice. Consulté le 27 septembre 2012, $<$ http://www.justice.gc.ca/fra/pr-rp/sjc-csj/harmonization/gaudr/fo/index.html>.

GÉmAR, Jean-Claude, dir. (1982): Langage du droit et traduction - Essais de jurilinguistique. Montréal: Conseil de la langue française.

GÉmAR, Jean-Claude (1983): Les trois états de la politique linguistique du Québec: d'une société traduite à une société d'expression. Québec: Conseil de la langue française.

GÉmAR, Jean-Claude (1995): Traduire ou l'art d'interpréter. Québec: Presses de l'Université du Québec.

GÉmAR, Jean-Claude (2006): Traduire la Common Law en français: possibilité du sens? Style et sens. In: Marianne Lederer, dir. Le sens en traduction. Paris/Caen: Lettres modernes Mignard, 153-159.

GÉMAR, Jean-Claude (2007): Style et sens du texte juridique en traduction. Un paysage choisi. Mélanges de linguistique française offerts à Leo Schena. Turin: L'Harmattan, 192-200.

GÉMAR, Jean-Claude (2011) : Traduire le droit. Lettre, esprit et équivalence. In: Marie CoRnu et Michel Moreau, dir. Traduction du droit et droit de la traduction. Paris: Dalloz, 129-144. 
GÉmAR, Jean-Claude et KAsIrer, Nicholas, dir. (2005): La jurilinguistique: entre langues et droits. Montréal/Bruxelles: Thémis/Bruylant.

GÉNY, François (1921): Science et technique en droit privé positif. Paris: Sirey.

Gouin, Jacques (1977): La traduction au Canada de 1791 à 1867. Meta. 22(1):26-32.

HART, Herbert L.A. (1994): The Concept of Law. $2^{\text {nd }}$ ed. Oxford: Clarendon Press.

Horguelin, Paul (1981): Anthologie de la manière de traduire. Montréal: Linguatech.

LABELLE, André (2000): La corédaction des lois fédérales au Canada. Vingt ans après: quelques réflexions. La traduction juridique. Histoire, théorie(s) et pratique. Genève: Université de Genève, 269-284.

Legrand, Pierre (2005): Issues in the Translatability of Law. In: Sandra Bermann et Michael Wood, dir. Nation, Language, and the Ethics of Translation. Princeton: Princeton University Press, 30-50

Loertscher, Denis (2001): La rédaction législative plurilingue dans le canton de Fribourg. LeGes. 2001(3):77-83.

Mattila, Heikki (2012): Jurilinguistique comparée. Cowansville: Éditions Yvon Blais.

Megale, Fabrizio (2008): Teorie della traduzione giuridica. Naples: Editoriale Scientifica.

Montesquieu (1758): De l'Esprit des loix. Genève: Barrillot \& Fils.

Mounin, Georges (1963): Les problèmes théoriques de la traduction. Paris: Gallimard.

Musso, Frédéric (2010): L’imparfait du fugitif. Paris: La Table ronde.

Nord, Christiane (2010): Text function and meaning in Skopos-oriented translation. In: Lewandowsка-Tомаszczyк, Barbara and Thelen, Marcel, dir. Meaning in Translation. Francfort: Peter Lang, 183-192.

NorD, Christiane (2004): Loyalität als ethisches Verhalten im Translationsprozess. In: Ina MüLLER, dir. Und sie bewegt sich doch... Translationswissenschaft in Ost und West, Festschrift für Heidemarie Salevsky zum 60. Geburtstag, Francfort: Peter Lang, 234-245.

Pigeon, Louis-Philippe (1982): La traduction juridique - L'équivalence fonctionnelle. In: JeanClaude GÉMAR, dir. Langage du droit et traduction - Essais de jurilinguistique. Montréal: Conseil de la langue française, 271-281.

Popovici, Adrian (2009): Libres propos sur la culture québécoise dans un monde qui rétrécit. McGill Law Journal/Revue de droit de McGill. 54(2):223-236.

Rigur, Paul (2004): Sur la traduction. Paris: Bayard.

SACCO, Rodolfo (2002): L'interprète et la règle de droit européenne. In: Rodolfo SACCo, dir. L'interprétation des textes juridiques dans plus d'une langue. Turin: L'Harmattan, 226-238.

SACCO, Rodolfo (2000) : Langue et droit. In: Erik JAY ME, dir. Langue et droit. Bruxelles: Bruylant, 224-260.

SACCo, Rodolfo (1999): Langue et droit. In: Rodolfo Sacco et Luca CAstellani, dir. Les multiples langues du droit européen uniforme. Turin: L'Harmattan, 163-185.

SARCEvic, Susan (2005): The Quest for Legislative Bilingualism and Multilingualism: Codrafting in Canada and Switzerland. In: Jean-Claude GÉmAr et Nicholas Kasirer, dir. La jurilinguistique: entre langues et droits. Montréal: Thémis, 277-292.

SARCEVIC, Susan (1997): New Approach to Legal Translation. La Haye: Kluwer.

Snow, Gérard et VAnderlinden, Jacques, dir. (1995): Français juridique et science du droit. Bruxelles: Bruylant.

SteIner, George (1992): After Babel. Oxford: Oxford University Press.

VANDERLINDEn, Jacques (1999): Contemporary Law. Canadian Reports to the 1998 International Congress of Comparative Law. Bristol/Cowansville: Éditions Yvon Blais, 25-68.

Villey, Michel (1975): Philosophie du droit. Paris: Dalloz.

VINAY, Jean-Paul (1978) : La traduction, une profession. In Proceedings of VIII ${ }^{\text {th }}$ world congress of the Fédération internationale des traducteurs (FIT), Montréal: Conseil des traducteurs et interprètes du Canada, 22.

Vinay, Jean-Paul et Darbelnet, Jean (1958): Stylistique comparée du français et de l'anglais. Montréal: Beauchemin

WiLss, Wolfram (2004): Translation Studies. The State of the Art. Meta. 49(4):777-785. 